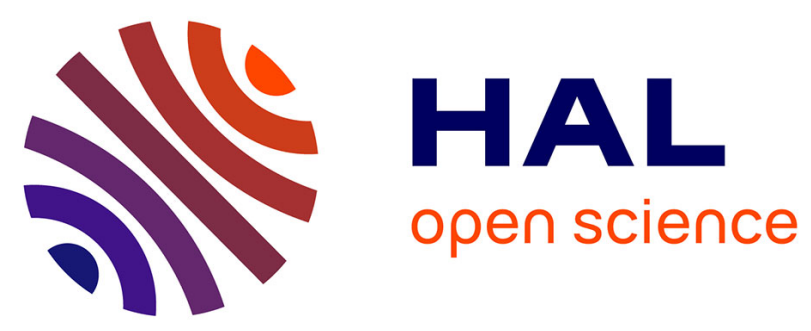

\title{
Towards a Blockchain Based Traceability Process: A Case Study from Pharma Industry
}

Ferdinando Chiacchio, Diego D'urso, Lucio Compagno, Marcello Chiarenza, Luca Velardita

\section{- To cite this version:}

Ferdinando Chiacchio, Diego D’urso, Lucio Compagno, Marcello Chiarenza, Luca Velardita. Towards a Blockchain Based Traceability Process: A Case Study from Pharma Industry. IFIP International Conference on Advances in Production Management Systems (APMS), Sep 2019, Austin, TX, United States. pp.451-457, 10.1007/978-3-030-30000-5_56 . hal-02419209

\section{HAL Id: hal-02419209 \\ https://hal.inria.fr/hal-02419209}

Submitted on 19 Dec 2019

HAL is a multi-disciplinary open access archive for the deposit and dissemination of scientific research documents, whether they are published or not. The documents may come from teaching and research institutions in France or abroad, or from public or private research centers.
L'archive ouverte pluridisciplinaire HAL, est destinée au dépôt et à la diffusion de documents scientifiques de niveau recherche, publiés ou non, émanant des établissements d'enseignement et de recherche français ou étrangers, des laboratoires publics ou privés.

\section{(c)(1)}

Distributed under a Creative Commons Attribution| 4.0 International License 


\title{
Towards a blockchain based traceability process: a case study from pharma industry
}

\author{
Ferdinando Chiacchio ${ }^{1}$, Diego D’Urso ${ }^{1}$, Lucio Compagno ${ }^{1}$, Marcello Chiarenza ${ }^{2}$ \& \\ Luca Velardita ${ }^{2}$ \\ ${ }^{1}$ University of Catania, Department of Electrical, Electronic \& Computer Engineering, Italy \\ ${ }^{2}$ SIFI $^{\circledR}$ SPA, Information \& Communication Technology, Lavinaio, Catania, Italy
}

\begin{abstract}
The technological boost brought by the world-wide program Industry 4.0 is leading the modernization of enterprises with solutions able to improve the automation of the factory. The pharmaceutical industry and the actors of the whole supply chain, adhering to strict regulations for ensuring the compliance to quality and safety standards, represents one of the most important stakeholders of the Industry 4.0 benefits.

In this paper, the serialization regulation and the technological solution adopted by an Italian pharmaceutical industry for the packaging lines of the factory is presented. As it will be shown, the conception of a traceability solution based on the blockchain technology is under investigation.
\end{abstract}

Keywords: Serialization, Vision Control Systems, Blockchain, SmartContracts.

\section{Introduction}

Industry 4.0 is a world-wide strategic initiative aiming to bring a technological revolution in the industry field [1]. By the mean of the well-known enabling technologies, production systems should be updated to an intelligent level, gaining the qualification of smart equipment. The factory should also be interconnected not only at a physical level but, and most of all, at the informative and automation level allowing to achieve a real-time communication and cooperation with humans, machines, sensors and so forth [2] in order to address a dynamic and global market [3].

To encourage this technology advancement, in these last years, many countries have destinated huge financing to industry. In Italy, this important initiative has taken place with the 2017 Budget Law that has established incentives, tax credits and benefits in relation to Industry 4.0 technologies, and investments in staff engaged in research and development (R\&D) [4].

In current years, Industry 4.0 solutions have been employed in the pharmaceutical industry under the regulation of several stakeholders to ensure safety and to protect the wellbeing of whole society [5]. In fact, pharmaceutical sector is a high-technology, knowledge-intensive and heavily regulated industry. All aspects of the life-cycle of new drugs are regulated, from patent application to marketing approval, commercial exploitation, patent expiration and competition with generics [6]. This applies to the 
other participants of the pharmaceutical supply chain, including distributors, healthcare providers, pharmacies, wholesalers, retailers and prescribing physicians who are subject to regulatory controls. This helps to ensure the product efficacy and safety which are not immediately observable [6].

This paper presents the solution that was applied for the serialization packaging process of SIFI ${ }^{\circledR}$ SPA, an Italian pharmaceutical industry, specialized in the preparation of ophthalmic products. As it will be shown, the serialization is the first of many improvements planned by the company which aim to modernize the factory with the enabling Industry 4.0 technologies. The conception of an improved traceability solution has been based on the blockchain technology.

The paper is organized as follows: in Section 2 the serialization regulation is presented. Section 3 gives a brief overview of the Company, illustrating the implemented serialization solution and the possible improvement of the traceability process based on blockchain technology. Finally, conclusions and future researches are depicted in Section 4.

\section{Serialization in pharma supply chain}

With the European Union's Directive 2011/62/EU, the European Parliament has provided a catalog of measures which are intended to prevent falsified medicinal products from entering the legal distribution chain. The 2011/62/EU Directive firmly regulates that, in the future, all prescription drugs must have safety labels that enable verification checks along the entire supply chain. Counterfeit drugs and falsified medicines [7] can then be identified immediately and safely withdrawn from circulation. The solution to this problem has been identified with the name of serialization [8]. Prerequisite for safeguarding against falsified medicines is the assignment of a unique serial number linked to the item production data (item identification code, expiry date, and batch number) in the form of a Data Matrix Code. Technology to automate the process of serial number generation, labelling and packaging is already in place in several pharmaceutical industries that are equipped with advanced machineries able to fully connect to the internet, realizing the IoT paradigm. In Italy, this process is being financed by the program Industry 4.0 [9].

Traceability is an essential key to safe drugs and the basis for absolute confidence on the part of the consumer in the pharmaceutical industry and its products. Figure 1 shows the supply process that involves several actors including the dispatching to the final customer. It is possible to notice that before any shipping, the Pharmaceutical Manufacturer has to communicate to a central regulatory (for instance, the European Medicines Verification Organisation in Europe, EMVO) all the serial numbers of drugs packages introduced into the distribution chain.

Safety, security, confidentiality, resilience and reliability related to the quality of the product and its transactions throughout the life-time and distribution chain must be guaranteed by the central regulatory although all the other actors (except the patient) are called to maintain for several years some of these information, in particular the one related to their individual responsibility. With this approach, any of these actors is 
responsible for its data and for the synchronization process with the regulatory that must behave as central authority.

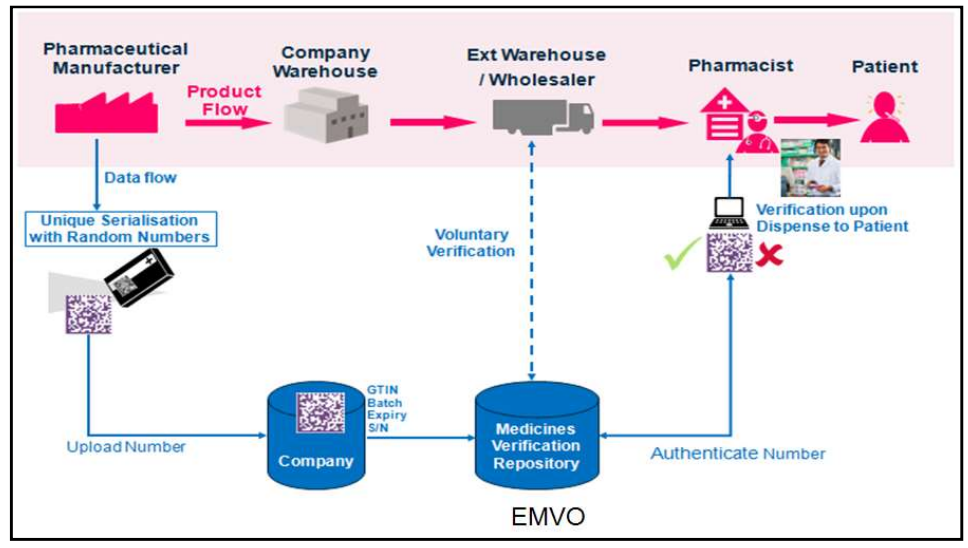

Fig. 1. Serialization and traceability

\section{Case of study: the SIFI SPA serialization process}

SIFI ${ }^{\circledR}$ SPA is a pharmaceutical company located in Lavinaio (CT), Italy, leader of the ophthalmic sector. SIFI ${ }^{\circledR}$ is also specialized in the production of medical and surgical devices mainly used in cataract surgery and diagnostic tools for ophthalmology. In this specific sector SIFI ${ }^{\circledR}$ boasts a modern and innovative production process and employs highly automated systems. With its products, SIFI ${ }^{\circledR}$ is present in the markets of Europe, Asia and America. Currently, SIFI ${ }^{\circledR}$ is engaged in a process of technology modernization in order to be compliant with the European Union's Directive 2011/62/EU and challenge the EU market, including Russia and Turkey.

The company promotes qualified scientific partnerships able to support this process and aims to extend and share with all the actors of the supply chain those technological initiatives which can bring to an improvement of the products quality for the final client.

\subsection{Packaging area layout description}

The packaging area is characterized by three different automated lines which serve respectively the single-dose vial, the multi-dose vial and the unguent/ointment products. More specifically, the single-dose and multi-dose vials lines are constituted by two packaging stages, the primary and the secondary. The machines of the first stage fill the primary vials (single or multi-dose) with the ophthalmic product, while the machines of the secondary stage arrange the final packaging boxes. To this end, the primary vials are placed inside their corresponding product box; different boxes are grouped into cases which will finally constitute the unit-load to be shipped.

The unguent/ointment production is independent and performs the whole packaging within the same line. 


\subsection{The serialization process}

The main objective of the serialization is to allow, at each level of the supply chain, the reconstruction of the packaging hierarchy of each box and the verification of the product quality. To achieve this goal and be compliant with the serialization specifications, each box must be marked with a unique identifier. The codification of the identifier must respect several rules that depends on the packaging level (Unit Box, Case Box, Unit-Load), on the product and, more important, on the encoding specification of the country in which the product will be commercialized.

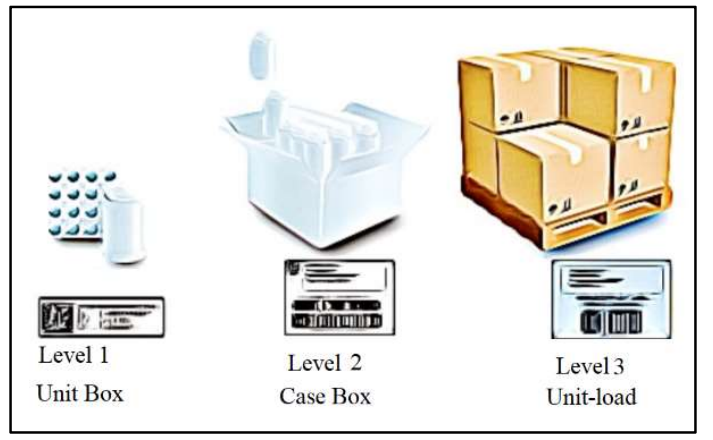

Fig. 2. Serialization and packaging hierarchy

As shown in Fig. 2, the marking of each box with a unique identifier allows to date back from the outer to the inner packaging level and vice versa, assuming that the packaging hierarchy is known and tracked in a computerized database. In fact, unitloads are not assembled randomly but according to the information carried by the work order. To this aim, the packaging process is initiated by the ERP that interacts with the packaging machines and with the vision system. This latter is a real-time system that, communicating to the printers of the packaging machines, verifies the correct marking of the identifier into the packaging boxes. Moreover, in the secondary packaging line, the vision system keeps track of the boxes that have been placed into each case and of the cases which have been placed into each unit-load. As soon the secondary packaging process has terminated, the serialization software sends the hierarchy matrix list to the ERP that closes the work order and elaborates the beginning of the next phases (storage, batch release, delivery/shipping). During the batch release, the serialization software sends to the central regulatory the official hierarchy matrix list which has to be stored permanently in the database of the serialization software.

To achieve the solution above described, many technological interventions have been realized exploiting the Industry 4.0 funding. A real-time control vision system has been installed and interfaced with the ERP by an ad-hoc customization of the serialization software. This latter is a multi-layer software solution constituted by several modules that act at all the levels of the manufacturing process (Figure 3). Moreover, the machines of the secondary packaging lines for both the single and multidose products (labeling machine, cartoning machine and palletizer) have been replaced 
with new equipment that can be interfaced with the factory informative system, adopting industrial standard communication protocols like OPC, ODBC and IOT.

\subsection{The Blockchain enhancement}

As discussed in Section 2, all the other actors of the supply chain are called to maintain for several years many information about the products. According to this principle, all the actors are responsible for its data and for the synchronization process with the regulatory that must behave as central authority.

Blockchain seems to offer a breakthrough in this important sector with a shared decentralized ledger resistant to tampering and able of preventing fraud [10]. It is not a case that further evolutions of the blockchain protocol are already being implemented $[11,12]$ and are becoming part of the business solutions offered by important players like IBM (Watson), SAP (Leonardo), DNV-GL, etc. Among them, smart contracts [13] represent the most promising blockchain application in business relationships. The main goal of a smart contract is to enable two anonymous parties to trade and do business with each other, usually over the internet, without the need for a middleman.

The main idea under investigation is to encrypt and store the hierarchy matrix list within a blockchain ledger. The process of serialization, including the blockchain enhancement, is shown in Figure 3.

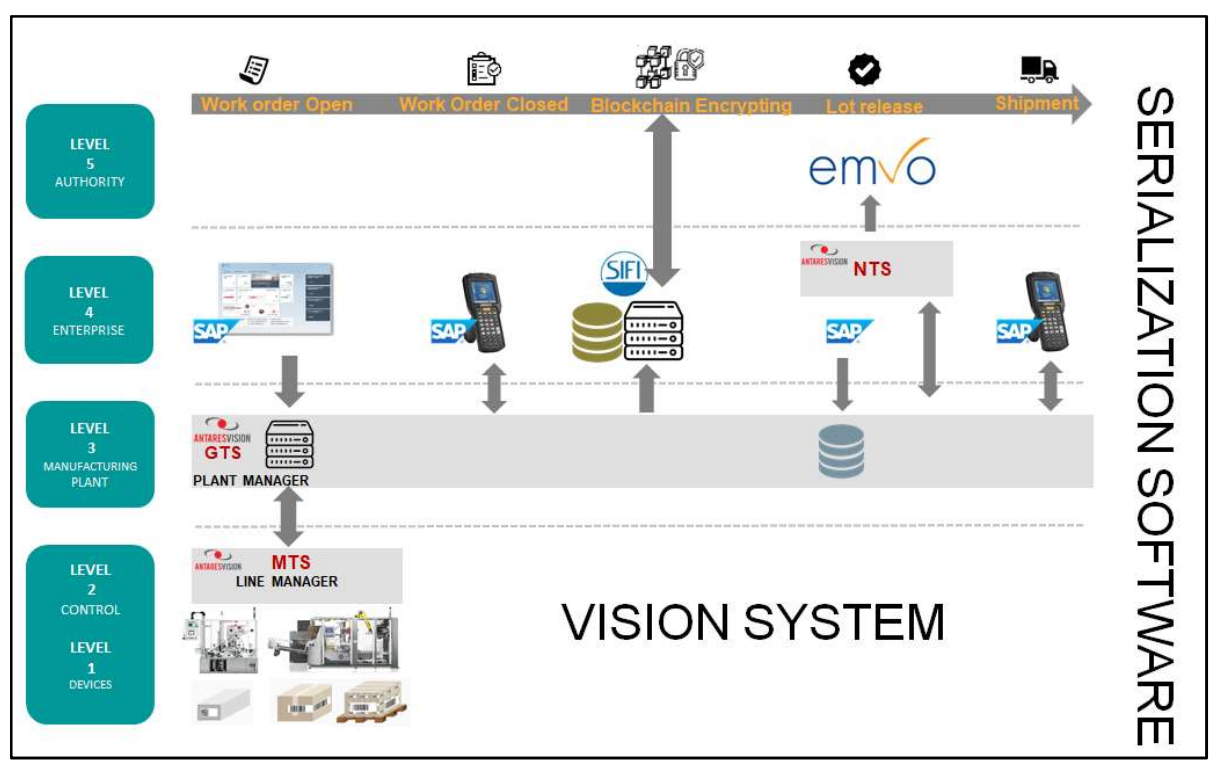

Fig. 3. Architecture of the serialization software and blockchain enhancement (courtesy of SIFI ${ }^{\circledR}$ )

After the work-order closure, the software of Level 3 (GTS Plant Manager) sends the hierarchy matrix list and related information to the enterprise server of Level 4 in charge to invoke the smart contract of the blockchain for storing and encrypting these data. In this way, the same information sent to the central regulatory will live 
permanently in the blockchain. Figure 4 shows the IT architecture (installed in the factory) that handle the traceability process via the blockchain network. In fact, data stored in the blockchain can be accessed by each actor of the traceability process and, to this end, ad-hoc Web3 HMI can be programmed.

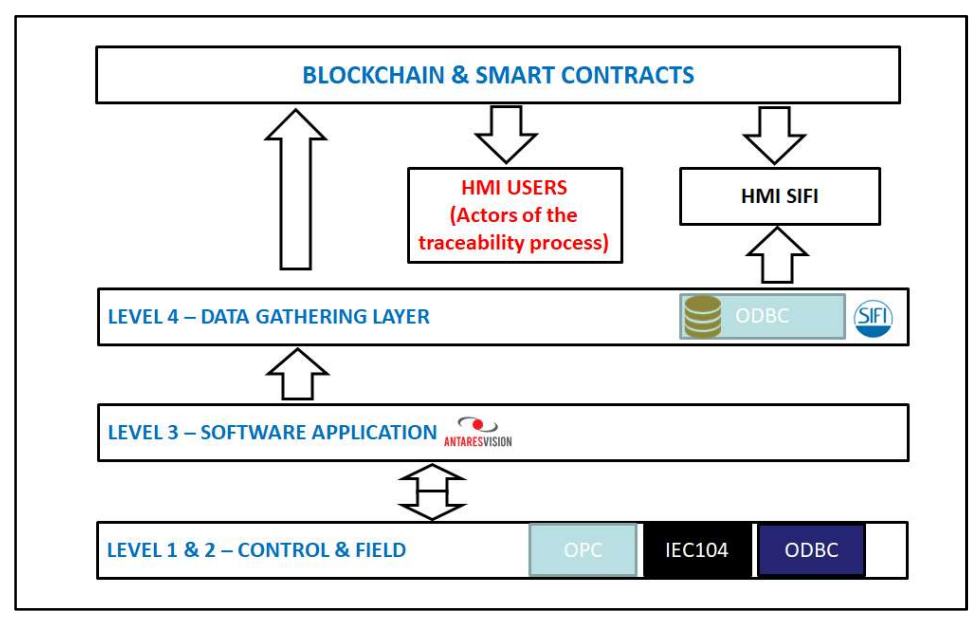

Fig. 4. IT architecture handling the traceability process via the blockchain network.

Further developments of the proposed application require the participation of the other actors of the supply chain. Exploiting the modern IT devices (nowadays fully connected to the internet), it is looks straightforward the possibility to implement an automated process that store into the blockchain the relevant information of the supplychain distribution process. In this way, together with the matrix list produced by SIFI ${ }^{\circledR}$, the actors of the traceability process can retrieve all this information and be guaranteed about the quality of the product along the supply chain. Moreover, exploiting these features, more complex B2B smart contracts [14] can be programmed in order to seal and verify the agreements among the various actors and guarantee, at any step of the distribution process, the compliance of the terms of contract.

\section{Conclusions and future researches}

In this paper, the serialization process of a pharmaceutical company has been illustrated. The main features of such regulation have been resumed and a case of study of an Italian industry was presented. The research collaboration with the company has brought to the conception of a blockchain solution for the serialization and traceability along the supply chain. To this end, the following activities will be carried on:

1 - Analyze the state-of-the-art of the commercial and open-source blockchain platform;

2 - Identify the main actors of the distribution process and the critical variables;

3 - Design a theoretical model of smart contract for the serialization process and the distribution chain; 
4 - Implement a prototype using an open-source blockchain platform.

\section{References}

1. Zhong RY, Xu X, Klotz E, Newman ST.: Intelligent Manufacturing in the Context of Industry 4.0: A Review. Engineering, Volume 3, Issue 5, October 2017, Pages 616-630.

2. Wang S, Wan J, Zhang D, Li D, Zhang, C. Towards smart factory for industry 4.0: a selforganized multi-agent system with big data based feedback and coordination. Computer Networks, (2016) 101, 158-168.

3. Shen, WM, Norrie DH: Agent-based systems for intelligent manufacturing: A state-of-theart survey. Knowledge Information Systems, an International Joural 1 (2) (1999), pp. 129156.

4. Seghezzi F, Tiraboschi M. Italy's Industry 4.0 Plan: An Analysis from a Labour Law Perspective. In: E-Journal of International and Comparative Labour Studies. - ISSN 22804056- 7 (1) (2018), pp. 1-29.

5. Ding B. Pharma Industry 4.0: Literature review and research opportunities in sustainable pharmaceutical supply chains. Process Safety and Environmental Protection, Volume 119, October 2018, Pages 115-130.

6. Sharma H, Parekh S, Shewale S. Regulatory Practice in Pharmaceutical Industry. Pharmaceutical Regulatory Affairs: Open Access. Volume 3, Issue 1, 2014

7. Smith G, Smith JA, Brindley DA. The Falsified Medicines Directive: How to secure your supply chain. Journal of Generic Medicines: Volume: 11 issue: 3-4, page(s): 169-172.

8. Whyte J. Pharmaceutical Serialization: An Implementation Guide, Global Serialization Lead, Rockwell Automation.

9. Circolare n. 4/E/2017 Agenzia Entrate - MiSE.

10. Mettler M. Blockchain technology in healthcare: The revolution starts here, e-Health Networking. In: IEEE 18th International Conference on Applications and Services (Healthcom), 2016.

11. Buterin V. Ethereum White Paper: A next generation smart contract \& decentralized application platform, https://www.ethereum.org/pdfs/EthereumWhitePaper.pdf

12. Huckle S, Bhattacharya R, White M, Beloff N. Internet of Things, Blockchain and Shared Economy Applications, Procedia Computer Science Volume 98, 2016, Pages 461-466.

13. Christidis K, Devetsikiotis M. Blockchains and Smart Contracts for the Internet of Things, Special Section in IEEE Access: The Plethora of Research in Internet of Things, 2016, pp: $2292-2303$.

14. Vechain. White Paper, https://www.vechain.org/ https://cdn.vechain.com/vechainthor_development_plan_and_whitepaper_en_v1.0.pdf 